\title{
Acoso Laboral (Mobbing): Riesgo Psicosocial Emergente Invisible
}

\author{
Adriana Marcela Manrique Torres ${ }^{1}$
}

Recibido: 25/10/2018 Aceptado: 26-08/2019

DOI: $10.21772 /$ ripo.v38n2a03

\begin{abstract}
Resumen
El acoso laboral o mobbing es considerado uno de los riesgos psicosociales emergentes, a pesar de la relevancia de esta problemática, aún se percibe la invisibilidad de este riesgo en las organizaciones. Este artículo de revisión presenta una perspectiva conceptual y teórica sumada a la exposición del vacío empírico y a las consecuencias del acoso laboral. En definitiva se encuentra que el acoso laboral se presenta como un proceso sistemático de desarrollo en la organización y persistente en el tiempo; así como la importancia de los perfiles de víctima / acosador y los factores organizacionales enmarcados en los factores de riesgo psicosocial intralaboral y riesgo psicosocial emergente, aun invisible para las organizaciones.
\end{abstract}

Palabras Clave: mobbing, riesgo psicosocial intralaboral, riesgo psicosocial emergente.

\section{Labor Harassment (Mobbing): Invisible Emerging Psychosocial Risk}

\begin{abstract}
Workplace bullying or mobbing is considered one of the emerging psychosocial risks, despite the relevance of this problem, the invisibility of this risk in organizations is still perceived. This review article presents a conceptual and theoretical perspective added to the empirical vacuum exposition and the consequences of workplace harassment. In short, it is found that labor harassment is presented as a systematic process of development in the organization and persistent over time; as well as the importance of the profiles of victim / stalker and the organizational factors framed in the factors of psychosocial risk intralaboral and emergent psychosocial risk, even invisible for the organizations.
\end{abstract}

Palabras Clave: mobbing, psychosocial risk intralaboral, emergent psychosocial risk.

1 Ms. En Psicología. Universidad Católica de Colombia. Docente Investigador. E-mail: psicologaocupacional@gmail.com

Cómo citar este artículo: Manrique Torres, A. M. (2019). Acoso Laboral (Mobbing): Riesgo Psicosocial Emergente Invisible. Revista Interamericana de Psicología Ocupacional, 38(2), 127-137. DOI: 10.21772/ripo.v38n2a03 


\section{Introducción}

La Organización Internacional del Trabajo [OIT] (2016) ratifica los factores de riesgo psicosocial como el eje causal del estrés, este enfoque establece que la interacción negativa entre los factores humanos y el medio ambiente del trabajo conlleva al desarrollo de afectaciones físicas o mentales. En este sentido, las actuales dinámicas de trabajo, los diversos modelos de organización y las demandas específicas en los diferentes roles laborales han complejizado los escenarios de trabajo de manera tal que la ambigüedad, el conflicto y el desempeño en el rol han potenciado la probabilidad de ocurrencia de los riesgos psicosociales, especialmente los intralaborales. Sumado a esto, contribuyen las figuras contractuales cambiantes, la ambigüedad e incertidumbre respecto a las condiciones de trabajo y la baja estabilidad laboral (Gil-Monte, 2012; Moreno, 2011).

En el marco de los riesgos psicosociales intralaborales, el acoso laboral o mobbing es considerado uno de los riesgos psicosociales emergentes. De acuerdo con Gil-Monte (2012) los riesgos psicosociales emergentes son fuentes o factores psicosociales que se generan por cambios organizativos o sociales o factores conocidos que se asumen como nuevos riesgos debido a percepciones sociales o avances científicos. Estos riesgos se han categorizado en cinco grupos: nuevas formas de contratación laboral, envejecimiento de la población laboral activa, retraso en la jubilación, intensificación del trabajo reflejado en la carga mental de la tarea, fuertes exigencias emocionales en el trabajo, aumento de acoso laboral y de violencia, finalmente, los riesgos asociados al desequilibrio y conflicto entre la vida laboral y personal, debido a la falta de ajuste entre la vida privada y las condiciones de trabajo. El presente artículo realiza una revisión de la literatura respecto el acoso laboral, también conocido con el anglicismo mobbing, el cual como riesgo psicosocial emergente parece no ser reconocido en las organizaciones, por tanto es invisibilizado, lo cual ha dificultado la implementación de mecanismos de identificación, prevención $\mathrm{y}$ gestión en las organizaciones. A continuación, se presenta una perspectiva conceptual y teórica; así como, algunos hallazgos empíricos y finalmente, las principales consecuencias del acoso laboral.

\section{Perspectiva Normativa y Conceptual de Acoso Laboral (Mobbing).}

A nivel supranacional de acuerdo con la Organización Mundial de la Salud [OMS] y la Organización Internacional del Trabajo [OIT] el acoso laboral es un problema de salud pública (Cassitto., et al 2004; Martínez et al., 2012; OMS, 2004; OIT; 2016). En el orden nacional a nivel normativo, para el caso de Colombia en el marco del Sistema de Gestión de Seguridad y Salud en el Trabajo se dispone la obligatoriedad de gestionar todos los riesgos laborales, sin excluir el riesgo psicosocial (Congreso de Colombia, 2012). En cuanto al riesgo psicosocial, específicamente, la resolución 2646 (M. Res. 2646, 2008), enfatiza en la necesidad de adelantar procesos de evaluación, investigación, gestión, intervención y monitoreo respecto los riesgos psicosociales en el trabajo para la determinación del origen de las patologías causadas por el estrés ocupacional (Ministerio de Protección Social, 2008). Así mismo, en 2006 se expide la resolución 1010 del Congreso de Colombia, conocida como la ley de acoso laboral que tiene por objeto definir, prevenir, corregir y sancionar las diversas formas de agresión, maltrato y toda vulneración a la dignidad humana que se ejerce sobre los trabajadores en el contexto de una relación laboral privada o pública. Esta ley enfatiza en que la conducta de acoso debe cumplir con dos condiciones de tipificación: persistencia y demostrabilidad; así mismo, establece como modalidades de acoso psicológico en el trabajo: la desprotección, inequidad, entorpecimiento, discriminación, persecución y maltrato laboral (Congreso de Colombia, 2006). 
En la línea de los riesgos psicosociales intralaborales se presenta la violencia laboral o violencia en el marco del trabajo con múltiples manifestaciones, una de ellas el acoso laboral o mobbing; en este sentido, "el terror psíquico o el acoso psicológico en la vida laboral significa una comunicación hostil y poco ética que se dirige de forma sistemática por una o varias personas principalmente hacia un individuo" (Leymann, 1990, p 120). Así mismo, de acuerdo con el meta-análisis de Topa, Depolo y Morales (2007) el acoso laboral no ha alcanzado una definición específica en consenso, pero se reconoce como un proceso de agresión sistemática y frecuente de actos negativos por parte de una persona o grupo hacia una persona en el ejercicio de la relación de poder como par, subordinado o superior en el trabajo, donde la persona vulnerada (víctima) posee una incapacidad para defenderse y se generan consecuencias graves, especialmente, a nivel psicológico; desafortunadamente la investigación práctica en esta problemática es menos robusta que a nivel teórico; cuyo vacío empírico se ha hecho evidente, específicamente, en Colombia (Londoño \& Orozco, 2015).

De acuerdo con Carvajal y Dávila (2013) el Mobbing es un tipo de violencia psicológica cuya raíz Mob como verbo indica "maltratar, asediar", utilizada por primera vez en 1963 por Konrad Lorenz, retomada en 1972 por Heinemann con relación a la conducta hostil entre los niños en edad escolar y desarrollado más ampliamente en 1980 por Leymann; de acuerdo con Leymann (1996, citado por Carvajal \& Dávila, 2013) el mobbing se caracteriza por conductas negativas, abusivas, hostiles, sofisticadas, cínicas y a la vez sutiles que se realizan de manera permanente y sistemática por una o más personas de la organización a fin de ofender, desprestigiar, humillar, excluir, estresar e interferir de manera negativa en sus actividades laborales con el objeto de obtener sus salida vulnerando su personalidad, integridad y dignidad (Carvajal \& Dávila, 2013).

\section{Metodología}

Este artículo de revisión expone un análisis teórico y conceptual de las variables consideradas en los principales modelos de acoso laboral; así como una reflexión sobre le vacío empírico manifiesto en este tema de alta sensibilidad.

\section{Algunos Modelos Teóricos de Abordaje del Acoso Laboral (Mobbing).}

En la línea de los modelos teóricos, uno de los modelos más conocidos es el modelo de Leymann (1990) conforme a este autor, el acoso laboral conocido como un fenómeno mediante el cual "se atrapa a alguien" o "terror psicológico" puede ser prevenido si se detecta a tiempo. En este sentido, de acuerdo con su modelo, la victimización se da por un proceso de cuatro fases: la primera caracterizada por el incidente crítico original donde el conflicto activa el proceso, la segunda donde se da el mobbing y la estigmatización y se desarrollan acciones que buscan ocasionar daño de manera sistemática, se caracteriza principalmente por la manipulación para afectar la reputación, comunicación y el desempeño de la víctima, la tercera donde la gestión de personal interviene y generalmente se asumen prejuicios sobre la víctima, esta fase se enmarca en el desarrollo legislativo que tenga cada país y generalmente requiere no sólo la valoración psicológica sino legal; y finalmente, la cuarta donde se da el momento de marginación o expulsión donde la victima abandona su trabajo y busca ayuda psicológica con efectos psicosomáticos y sociales. Teniendo en cuenta que en el acoso laboral se presenta vulneración a los derechos laborales, de acuerdo con el autor, deben desarrollarse métodos de rehabilitación complementarios a nivel social y legal.

De acuerdo con el modelo de Leymann el acoso debe ser persistente (por lo menos una vez a la semana) y prolongado (por lo menos durante 
seis meses), lo anterior para tipificar la conducta en frecuencia, duración e intensidad a fin de determinar el diagnóstico del mobbing.

En relación con lo anterior, Einarsen (2000, citado por Carvajal \& Dávila, 2013) identificó tres modelos desde los cuales identificar causas del mobbing: en función de la personalidad de la víctima y el acosador, el clima organizacional y en función de la interlocución e interacción humana. En las causas de la organización se encuentran la mayoría de factores de riesgo psicosocial; tales como: deficiente liderazgo, insatisfacción del clima, inadecuado diseño de la tarea, problemas de comunicación y cultura organizacional; entre otros. En los efectos se puede resaltar desesperación, ira, ansiedad, depresión, melancolía apatía, agresividad, fobia social, falta de concentración, estrés, trastorno del sueño, estrés post-traumático, trastornos psicosomáticos, problemas psicológicos y suicidio; también se pueden apreciar consecuencias en el entorno social, familiar y su adaptación al contexto; además, con relación a la organización aumenta el ausentismo y la rotación, disminuye la productividad, el compromiso, la satisfacción, y aumenta la intención de abandonar el puesto de trabajo. Al mismo tiempo, desde la interacción se puede apreciar el acoso laboral desde la rivalidad, la competencia permanente, las coaliciones entre compañeros con relación a reforzar el maltrato hacia el acosado, la negación de la existencia del acoso en el ambiente y por consecuencia la negación de ayuda a las víctimas de la organización según lo reportan Hirigoyen y Luna (2001, 2003; citados por Carvajal \& Dávila, 2013).

Con relación al perfil, según Martínez et al. (2012) la persona vulnerada o víctima y el acosador podrían estar enmarcados en características distintivas; tales como: en la víctima, generalmente mujeres entre 35 y 45 años, con nivel de auto-exigencia elevado, autónomos, alto sentido de moralidad y respeto por las normas sociales, destacados profesionalmente, con preocupación por la autoimagen; así mismo, en el acosador, se puede encontrar: cobardía, manipulación, culpabilidad, agresividad y un mediano desempeño.

Por otro lado, Ege (2000), indica que aunque comprender el acoso laboral es complejo, lo identifica como un goteo de humillación, ataque y persecución donde los actores que interactúan son los principales movilizadores del fenómeno, tanto víctima como acosador; así como, sus concepciones, valores, idiosincrasia y personalidad; así mismo, involucra la dimensión cultural y contextual del comportamiento acosador y amplía las cuatro etapas planteadas por Leymann a seis, contemplando una etapa previa denominada "condición cero" donde aparece la fase inicial del conflicto, el cual no se destruye sino que sobre sale y posteriormente se desarrollan las seis etapas planteadas: 1) conflicto objetivo, 2) aparición del mobbing ( si las conductas de acoso se convierten en un hábito y son sistemáticas a largo plazo se puede aludir acoso psicológico), 3) primeros síntomas psicosomáticos, 4) errores y abuso en la gestión del talento humano, 5) decaimiento de la condición psicofísica individual caracterizada por desesperanza y desesperación con o sin comorbilidad con ansiedad y depresión, y finalmente, 6) exclusión del trabajo por despido, renuncia o en el peor de los casos suicidio. El autor identifica una fase final adicional, específicamente denominado doble mobbing, donde la familia de la víctima se ve de alguna manera afectada por el acoso laboral, aunque nunca de la misma manera que la víctima.

Con referencia a los modelos, de acuerdo con Flores, Tovar y Vilchis (2014) se han desarrollado modelos de explicación del acoso laboral o mobbing desde 1962, con enfoque organizativo; entre estos: análisis de factores organizacionales, medidas de higiene y seguridad, entornos de trabajo arriesgados, competencia y 
cambio tecnológico, reestructuración (poder), reducción de costos, productividad, clima y gestión, ausencia de liderazgo, estilos autoritarios o débiles de gestión, estrés (caracterizado por ambientes negativos), perfiles de trabajo y ambigüedad en el rol, factores estacionales (alta frecuencia), comportamiento diverso, conductas hostiles.

Además, otros modelos con objeto de estudio en variables particulares de algún otro orden: en 1962 el modelo de Langner, basado en la desacreditación, en 1972 el modelo de Goldberg, basado en la inseguridad y estrés, en 1994 el modelo de López, Hoel y Zapf, centrado en rasgos de la personalidad y características de la organización, en 1996 el modelo de Leymann, centrado en la restricción de la comunicación y la desacreditación, en 1997 el modelo de Einarsen, Raknes y Matthiesen, centrado en los estilos de liderazgo, en el año 2000 el modelo de Quine, centrado en factores jerárquicos, situacionales y de identidad, en 2001 el modelo de Piñuel y Zavala, centrado en factores situacionales, organizacionales y de comportamiento organizacional, en 2001 el modelo de Einarsen y Hoel, centrado en los perfiles psicológicos y la frecuencia del acoso, en 2003 el modelo de Knorz y Zapt, basado en las habilidades sociales y la temporalidad, en 2004 el modelo de Bjorkqvist, Osterman y Lagerspetz, que integra la variable perspectiva de género, en 2004 el modelo Van Dick y Wagner, quienes realizan una adaptación del instrumento de Leymann y se incluyen los estilos de gestión; así como, en 2005 el modelo de González de Rivera que también utiliza el instrumento de Leymann e incluye factores organizativos y perfiles psicológicos (Flores, Tovar \& Vilchis, 2014).
En consecuencia, de acuerdo con Flores, Tovar y Vilchis, (2014) el modelo teórico de acoso laboral incluye los siguientes elementos: factores de la organización, factores de conflicto que involucran la personalidad de la víctima y el acosador abarcando el componente de la inteligencia emocional; así como, factores situacionales e identidad cultural. Estos autores reconocen que existe diversidad de variables con relación a la medición del constructo mobbing, e indican que es evidente la falta de integración de la variable inteligencia emocional en los modelos ya conocidos. En este sentido, indican no sólo una alta correlación de esta variable (inteligencia emocional) con el constructo mobbing; sino, que hacen referencia a la falta de inclusión de la perspectiva de género en la investigación del mobbing en general e indican alta incidencia del mobbing en educación superior, particularmente en un contexto mexicano; así como, en organizaciones cerradas con alta valoración del control y el poder (Flores, Tovar, González \& Hernández, 2008; Flores, Tovar \& Vilchis, 2014).

Por otra parte, Diaz-Garcia (2018) plantea el contexto público como un entorno facilitador de las condiciones que propician el acoso laboral, este autor hace un análisis del contexto Español frente al poder y el liderazgo, concluye que existen elementos predictores del acoso laboral relacionados con las relaciones asimétricas de poder y los estilos de liderazgo que son ejercidos en los contextos laborales. De la misma manera, para Cornoiu y Gyorgy (2013) identificar el fenómeno del acoso laboral a tiempo en la organización constituye uno de los mejores avances en prevención, en tanto se reconoce que existe una problemática que requiere abordaje inmediato y oportuno. 
Tabla 1. Principales Modelos de Acoso Laboral (Mobbing)

\begin{tabular}{|c|c|c|}
\hline Leymann (1990) & Ege (2000) & Einarsen (2000) \\
\hline Desarrollo Acoso Laboral en Fases: & $\begin{array}{l}\text { Aumenta fases al Modelo } \\
\text { de Leymann }\end{array}$ & $\begin{array}{l}\text { Desarrollo del Acoso Laboral a partir de } \\
\text { tres condiciones }\end{array}$ \\
\hline 1. Incidente Crítico o Conflicto. & 0. Condición cero. & 1. Personalidad de la Víctima: Perfil \\
\hline $\begin{array}{l}\text { 2. Daño Sistemático: } \\
\text { 2.1 Restricción de la comunicación. } \\
\text { 2.2 Desempeño. }\end{array}$ & 1. Conflicto objetivo. & $\begin{array}{l}\text { 2. Clima Organizacional: Factores de } \\
\text { Riesgo Psicosocial. }\end{array}$ \\
\hline $\begin{array}{l}\text { 3. Intervención de Talento Humano. } \\
\text { 3.1 Valoración Psicológica. } \\
\text { 3.2 Valoración legal. }\end{array}$ & 2. Aparición del mobbing. & $\begin{array}{l}\text { 3. Características de la comunicación: } \\
\text { Interlocución e interacción humana. }\end{array}$ \\
\hline \multirow[t]{5}{*}{$\begin{array}{l}\text { 4. Marginación, expulsión o } \\
\text { abandono de trabajo por la Víctima. }\end{array}$} & $\begin{array}{l}\text { 3. Primeros síntomas } \\
\text { psicosomáticos. }\end{array}$ & \\
\hline & $\begin{array}{l}\text { 4. Errores y abuso en la gestión } \\
\text { del talento humano. }\end{array}$ & \\
\hline & $\begin{array}{l}\text { 5. Decaimiento de la condición } \\
\text { psicofísica. }\end{array}$ & \\
\hline & 6. Exclusión del trabajo & \\
\hline & 7. Doble mobbing & \\
\hline
\end{tabular}

Nota: Elaboración Propia.

Tabla 2. Algunos Modelos Recientes de Acoso Laboral (Mobbing)

\begin{tabular}{lll}
\hline \multicolumn{1}{c}{ Martínez et al. (2012) } & \multicolumn{1}{c}{ Flores, Tovar y Vilchis (2014) } & Díaz-García (2018) \\
\hline Importancia de los actores: & Tres Factores: & Predictores: \\
\hline 1. Perfil Víctima. & $\begin{array}{l}\text { 1. Factores de la Organización: Factores de } \\
\text { Riesgo Psicosocial. }\end{array}$ & 1. Contexto Laboral Público \\
& 2. Factores del Conflicto: Personalidad, & 2. Relaciones asimétricas de poder \\
2. Perfil Acosador. & Víctima y Acosador (Inteligencia & \\
\hline
\end{tabular}

3. Factores Situacionales: Identidad Cultural 3. Estilos de liderazgo.

Nota: Elaboración Propia.

\section{Algunos Hallazgos Empíricos}

Un estudio buscó analizar la relación entre percepciones de mobbing y autoeficacia de los docentes respecto la percepción de los funcionarios escolares. El estudio establece como antecedente la importancia de identificar que los conflictos no resueltos entre los trabajadores generan conflicto organizacional que conducirán al desarrollo de estrés y acoso laboral o mobbing; enmarca la vulnerabilidad de los contextos educativos debido a que la generalidad de las condiciones de desarrollo de la profesión de los educadores conlleva a riesgos respecto la salud mental, este estudio con una muestra de 412 personas encontró que las percepciones de mobbing varían respecto variables como: edad (23-32 y 33-42 años, mayor edad, mayor riesgo), sexo, tipo de trabajo, años en 
la misma institución educativa (6 a 10 años), años bajo la misma administración (6 a 10 años); así mismo, los maestros que tienen una vinculación por contrato son más vulnerables al acoso que quiénes tienen una vinculación laboral directa: de igual manera, se encontró relación entre la baja percepción de autoeficacia y la percepción de mobbing encontrando que prima el acoso laboral aplicado entre colegas respecto el ejercido en otro tipo de relaciones laborales, este estudio encontró que el mobbing afecta negativamente la autoeficacia del docente al parecer porque las personas se sienten psicológicamente agotadas y han perdido su capacidad profesional (Celep \& Eminoglu, 2012).

Al mismo tiempo, Aydan, Mustafa y Tuna (2012) identificaron conductas percibidas como mobbing en 280 instructores de educación especial, donde se identificó que los comportamientos de mobbing se desarrollan principalmente en contexto laborales, con mayor frecuencia por parte de los gerentes, se asocian generalmente con falta de reconocimiento por el desempeño laboral, aumento de carga laboral, falta de justicia organizacional, evaluación de desempeño inequitativa, aumento de responsabilidad y disminución de la autoridad; así mismo, identificaron mayor percepción de acoso laboral en los rangos de menor edad respecto a las superiores. Igualmente, otro estudio con 238 empleados de salud identificó una relación estadísticamente significativa entre exposición al mobbing y satisfacción con la profesión; así mismo, algunas áreas de trabajo como emergencias, cuidados intensivos y cirugía se asociaron más con la experiencia de mobbing (Görgülü, Beydağ, Şensoy \& Kiyak, 2014).

Por otra parte, un estudio descriptivo de Rivera, Martínez y Cox (2014) indicó en una muestra de 150 empleados en maquiladoras del norte de México que al menos 138; es decir el $92 \%$ indicaron haber experimentado por lo menos uno de los comportamientos de acoso laboral o mobbing; así mismo, el 8\% manifestaron experimentar un alto grado de acoso laboral. De igual forma, indicaron haber experimentado al menos un incidente de acoso en los últimos seis meses, así como el liderazgo y el poder ejercido como condiciones facilitadoras del acoso laboral.

De igual manera, Ibarra, Escalante y Mendizábal (2015) identificaron acoso laboral en el ámbito de la docencia, especialmente en ambientes de educación superior; así mismo, corroboraron afectaciones psicológicas, físicas, familiares y sociales; lo anterior, enfatiza la necesidad de prevención desde los diferentes niveles y frentes de manifestación. En esta misma línea descriptiva, Guimarães, Cançado y Lima (2016) caracterizaron un caso de acoso laboral en una institución de educación superior mediante el análisis documental del proceso administrativo; así como, una entrevista de profundidad con la victima donde se encontró un caso de acoso basado en el abuso de poder conduciendo a graves consecuencias de salud a nivel físico y mental. Igualmente, el análisis de caso identificó la falta de intervención del área de talento humano tanto en el desarrollo del conflicto, como en la cultura de la organización.

Un meta-análisis analizó 36 muestras independientes de 13.896 personas y 101 medidas de tamaño de efecto, con el fin de establecer la relación existente entre la exposición al acoso y el modelo de cinco factores de personalidad, encontrando que la exposición al acoso laboral se asocia positivamente con el rasgo del neuroticismo, negativamente con la extraversión, no se relaciona con la apertura y es significativo con la amabilidad y meticulosidad. En este sentido, el estudio pone de manifiesto la importancia de considerar las condiciones personales en el acoso toda vez que hay una relación entre los rasgos de personalidad y la exposición al acoso laboral; de esta manera se corrobora primordialmente el rasgo de neuroticismo como rasgo referenciado 
en algunos modelos teóricos de acoso (Nielsen, Glasø \& Einarsen, 2017).

Asimismo, otro estudio buscó identificar los niveles de intimidación percibida y establecer asociaciones con síntomas psicológicos experimentados, las personas divorciadas, jóvenes y con enfermedad crónica percibían mayor intimidación en el lugar de trabajo, estas percepciones se correlacionaron con puntajes altos en somatización, hostilidad, ansiedad y depresión. Con respecto a la variable de mayor correlación con la percepción de hostilidad fue la edad en el grupo de 20 a 29 años, a menor edad mayor percepción de acoso laboral (Duru, Ocaktan, Çelen \& Örsal, 2017).

\section{Consecuencias del Acoso Laboral}

De acuerdo con Leymann (1990) llama la atención a nivel psicosomático los síntomas de depresión, compulsión, suicidio; a nivel psicológico ansiedad y pérdida de estrategias de afrontamiento; así como, a nivel social desempleo voluntario, estigmatización y aislamiento social. Otras condiciones sociales que se resaltan son: largos periodos de incapacidad, expulsión, aislamiento social, desempleo e inicio de procesos de intervención a nivel psiquiátrico o psicológico.

Por otra parte, Martínez et al. (2012) enfatiza que las consecuencias para la víctima se dan a nivel psicológico, principalmente por síntomas de estrés y estrés crónico que genera la intimidación de manera sistemática y permanente; a nivel físico por la somatización que puede generar trastornos funcionales y orgánicos; así como, síntomas psicosomáticos, a nivel social desconfianza, hipersensibilidad a la crítica, sentimientos de ira y deseo de venganza; al igual que conductas de evitación, aislamiento y hostilidad.

De acuerdo con Ege (2000) los estados depresivos y ansiosos a causa de la tensión permanente e incontrolada generan daño psicológico y afecto, primordialmente la autoconfianza, el interés, la dignidad, el entusiasmo en el trabajo, la productividad, la influencia en el trabajo, la capacidad de tomar decisiones y el respeto por los demás. Al mismo tiempo, el autor resalta el aislamiento, como uno de los principales síntomas de la persona acosada.

\section{Invisibilidad del Mobbing en las Organizaciones}

De acuerdo con la asociación colombiana de psiquiatría (2012, citado por el Ministerio de Trabajo, 2016) en el protocolo de prevención y actuación de acoso laboral, para el año 2012 se estimó que uno de cada cinco trabajadores del país eran víctimas de algún tipo de acoso laboral; de igual manera, para el año 2013 se presentaron 361 denuncias por acoso laboral y se considera en aumento significativo (Ministerio de Trabajo, 2016). En esta línea de acuerdo con el ministerio de trabajo las acciones preventivas de acoso laboral giran en torno a las políticas, acciones de psicoeducación, implementación de normas de convivencia y desarrollo de comités de convivencia; de igual forma, las acciones correctivas deben darse con relación a la intervención de factores de riesgo psicosocial y los procedimientos para corregir las conductas de acoso laboral (Ministerio de Trabajo, 2016).

Conviene subrayar que dada la complejidad del fenómeno, la estrecha relación con variables de clima, cultura, liderazgo y riesgo psicosocial intralaboral, al igual que la falta de acceso a la información de los trabajadores, el temor a la pérdida del trabajo, la ausencia de solidez en los procesos de intervención y seguimiento; así como, la misma invisibilidad del fenómeno desde las organizaciones ha dificultado no solo su intervención y prevención, sino la investigación del mismo en contextos diversos de ocupación $\mathrm{y}$ en el marco preventivo del sistema general de 
riesgos laborales. Esta noción es apoyada por otros autores con énfasis en el seguimiento en cuanto a la falta de garantías y mecanismos de orden particular y específico para la vigilancia y control legal a nivel preventivo (Londoño \& Orozco, 2015).

\section{Conclusiones}

La mayoría de modelos presentados en este artículo consideran consecuencias para las víctimas de tipo psicológico, psicosomático y de orden social (Ege, 2000; Leymann, 1990; Martínez et al., 2012). Asimismo, algunos autores coinciden en la importancia de contemplar en el acoso laboral las condiciones específicas de los perfiles de víctima y acosador (Ege, 2000; Einarsen, 2000; Flores, Tovar \& Vilchis, 2014; Martínez et al., 2012).

El acoso laboral o mobbing es un riesgo psicosocial emergente que presenta un vacío empírico y aún no posee suficiente investigación, específicamente en Colombia. Actualmente se enmarca en un escenario de protección a nivel supranacional y nacional que implica la gestión de los riesgos laborales, incluyendo el riesgo psicosocial. En este sentido, desconocer de qué forma las fuentes generadoras de riesgo psicosocial intralaboral y los riesgos psicosociales emergentes contribuyen a afectaciones psicológicas, psicosomáticas y sociales en los trabajadores genera a nivel práctico que los programas de intervención en riesgo psicosocial en el marco del trabajo estén desarticulados de los contextos específicos de ocupación y de su relación con la gestión directa de la fuente de riesgo y riesgo emergente que consolida estas afectaciones psicosociales y disminuye la salud en general y la calidad de vida laboral (De Jesús et al., 2016; Gil-Monte, 2012; Moreno, 2011).

Desde el enfoque de gestión del riesgo se deben eliminar o gestionar las fuentes de riesgo para potencializar los niveles de prevención de los mismos, los riesgos psicosociales emergentes implican tensión psicológica permanente para todos los individuos, la exposición permanente y crónica involucra también un problema de salud laboral y salud pública, más allá del coste económico y social implícito (Gil-Monte, 2012). En esta línea, la satisfacción laboral de los trabajadores afecta su motivación laboral, la calidad de trabajo y el desgaste emocional. De esta manera, si se previene el acoso laboral, se interviene la calidad de vida en el trabajo y la satisfacción con el mismo, aspectos apoyados a nivel empírico (Görgülü, Beydağ, Şensoy \& Kiyak, 2014). Con respecto a lo anterior, en relación a los elementos de poder y liderazgo en la organización, la prevención primaria en la organización con relación al acoso laboral estarían dados por otras variables que amplían el espectro y centran la gestión en procesos específicos: la habilidad de gestión, refuerzo, supervisión constructiva, justicia organizacional, bienestar, desarrollo de motivación y capital psicológico que consolida un liderazgo preventivo y adecuado (Diaz-Garcia, 2018).

A pesar de los múltiples modelos de acoso laboral y de las diversas variables que pueden ser contemplados como predictores, se aprecian algunos elementos trasversales que coinciden en los modelos más sólidos presentados aquí: el acoso laboral como un proceso sistemático de desarrollo en la organización y persistente en el tiempo, el impacto de los perfiles de víctima y acosador, finalmente; los factores organizacionales enmarcados en los factores de riesgo psicosocial y riesgo psicosocial emergente. La complejidad e invisibilidad del fenómeno incide en una carencia de prevención y gestión del mismo en el marco del sistema general de riesgos laborales, aún falta mayor alcance en el seguimiento, sistematización del comportamiento epidemiológico del fenómeno e investigación al respecto; en muchas ocasiones, los trabajadores 
terminan abandonando su trabajo y no solo las organizaciones presentan fugas de talentos, sino que el coste social desde lo individual trasciende el grupo familiar, lo que lo convierte en un riesgo psicosocial emergente invisibilizado de amplio y complejo alcance.

\section{Referencias}

Aydan, A., Mustafa, O., \& Tuna, S. (2012). Behaviors perceived as mobbing by the instructors assigned in special education institutions. Procedia-Social and Behavioral Sciences, 46, 4858-4863.

Cassitto, M. G., Fattorini, E., Gilioli, R., Gonik, V., Fingerhut, M. A., Kortum-Margot, E. G., \& World Health Organization. (2004). Sensibilizando sobre el acoso psicológico en el trabajo. Recuperado de: http://bit. ly/2DHJILY

Carvajal, J., \& Dávila, C. (2013). Mobbing o acoso laboral. Revisión del tema en Colombia. Cuadernos de Administración, 29 (49), 95-106.

Celep, C., \& Eminoglu, E. (2012). Primary school teacher's experience with mobbing and teacher's self-efficacy perceptions. Procedia-Social and Behavioral Sciences, 46, 4761-4774. https://doi.org/10.1016/j. sbspro.2012.06.332

Colombia. Congreso de la República. (2006) Ley 1010 de 2006. Por medio de la cual se adoptan medidas para prevenir, corregir y sancionar el acoso laboral y otros hostigamientos en el marco de las relaciones de trabajo. Bogotá: Autor.

Colombia. Congreso de la República. (2012) Ley 1562 de 2012. Por la cual se modifica el Sistema de Riesgos Laborales y se dictan otras disposiciones en materia de salud ocupacional. Bogotá: Autor.

Colombia. Ministerio de Trabajo. (2016) Protocolo de Prevención y Actuación de
Acoso Laboral. Bogotá, Colombia.

Colombia. Ministerio Protección Social. (2008). Resolución 2646. Por la cual se establecen disposiciones y se definen responsabilidades para la identificación, evaluación, prevención, intervención y monitoreo permanente de la exposición a factores de riesgo psicosocial en el trabajo y para la determinación del origen de las patologías causadas por el estrés ocupacional. Bogotá: Autor.

Cornoiu, T. S., \& Gyorgy, M. (2013). Mobbing in Organizations. Benefits of Identifying the Phenomenon. Procedia-Social and Behavioral Sciences, 78, 708-712.

De Jesús, B. M., da Silva, S. R., Carreiro, D. L., Coutinho, L. T. M., Santos, C. A., de Barros Lima, A. M. E., \& Coutinho, W. L. M. (2016). Relação entre Síndrome de Burnout e Condições de Saúde entre Militares do Exército. [Relación entre Síndrome de Burnout y Condiciones de Salud entre Militares del Ejército]. Tempus Actas de Saúde Coletiva, 10(2), 11-28. doi.org/10.18569/tempus.v10i2.1606.

Díaz-García, O. (2018). Bullying in Public Administration. In Farazmand, A. (ed.), Global Encyclopedia of Public Administration, Public Policy, and Governance. Shringer, Cham (Switzerland), pp. 1-9.

Duru, P., Ocaktan, M. E., Çelen, Ü., \& Örsal, Ö. (2017). The Effect of Workplace Bullying Perception on Psychological Symptoms: A Structural Equation Approach. Safety and Health at Work. http://dx.doi. org/10.1016/j.shaw.2017.06.010

Ege, H. (2000). Mobbing: Il Terrore Psicologico sul Posto di Lavoro e le sue conseguenze Psicofisiche Sull'individuo. Leadership Medica, 3C 
Flores, M. M. T., Tovar, L. A. R., González, J. I. R., \& Hernández, J. A. G. (2008). Propuesta de un modelo de mobbing bajo la óptica administrativa, utilizando en sus variables la inteligencia emocional. Criterio Libre, (9), 139-161.

Flores, M. M. T., Tovar, L. A. R., \& Vilchis, F. L. (2014). Mobbing: A Theoretical Model Quantifying Factors Affecting the Role of Women Executives in The Institutions of Public Education in Mexico. Contaduría y Administración, 59(1), 195-228.

Gil-Monte, P. R. (2012). Riesgos Psicosociales en el Trabajo y Salud Ocupacional. Revista Peruana de Medicina Experimental y Salud pública, 29(2), 237-241.

Görgülü, N., Beydağ, K. D., Şensoy, F., \& Kiyak, M. (2014). The Effects of Mobbing (Bullying) on Health Employes. ProcediaSocial and Behavioral Sciences, 152, 503509.

Guimarães, C. A., Cançado, V. L., \& Lima, R. D. J. C. (2016). Workplace moral harassment and its consequences: A case study in a federal higher education institution. Revista de Administração, 51(2), 151164. doi: 10.5700/rausp1231

Ibarra, L. M., Escalante, A. E., \& Mendizábal, G. (2015). El acoso laboral entre los trabajadores universitarios. RICSH Revista Iberoamericana de las Ciencias Sociales y Humanísticas, 4(7).

Leymann, H. (1990). Mobbing and psychological terror at workplaces. Violence and Victims, 5(2), 119-126.
Londoño, C. A. D., \& Orozco, J. G. C. (2015). Aproximación al estado actual de la prevención del acoso laboral en Colombia. Sotavento MBA, (26), 10-25.

Martínez, M. D. L. M., Irurtia, M. J., Martínez, C., Torres, H., \& Queipo, D. (2012). El Acoso Psicológico en el Trabajo o Mobbing: Patología Emergente. Gaceta Internacional de Ciencias Forenses, $\mathrm{n}^{\mathrm{o}} 3$. Abril-Junio: 5-12

Moreno, B. (2011). Factores y riesgos laborales psicosociales: conceptualización, historia y cambios actuales. Medicina y Seguridad del Trabajo, 57(Supl. 1), 4-19. https://dx.doi.org/10.4321/S0465546X2011000500002

Nielsen, M. B., Glasø, L., \& Einarsen, S. (2017). Exposure to workplace harassment and the Five Factor Model of personality: A meta-analysis. Personality and Individual Differences, 104, 195-206. https://doi. org/10.1016/j.paid.2016.08.015.

Organización Internacional del Trabajo OIT (2016). Estrés en el Trabajo: Un reto colectivo. Ginebra, Autor.

Rivera, B. R. G., Martínez, I. A. M., \& Cox, J. L. (2014). The hidden face of mobbing behavior Survey application of the Cisneros inventory in a maquila facility in Mexico. Contaduría y Administración, 59(1), 175-193.

Topa, G., Depolo, M., \& Morales, J. F. (2007). Acoso laboral: meta-análisis y modelo integrador de sus antecedentes $\mathrm{y}$ consecuencias. Psicothema, 19(1). 